James P. McCormack Pharm D, ${ }^{*} \dagger$ C. Brian Warriner MD, $\ddagger$ Marc Levine PhD, ${ }^{*}$ Ned Glick PhD§

\title{
A comparison of regularly dosed oral morphine and on- demand intramuscular morphine in the treatment of postsurgical pain
}

$A$ randomized, placebo-controlled, double-blind clinical trial was conducted to compare the use of regularly dosed po morphine and on-demand im morphine in 47 patients undergoing total hip arthroplasty. Patients were randomized to receive either $20 \mathrm{mg}$ (initial dose) of regularly dosed morphine (every four hours po) plus breakthrough pain medication on-demand consisting of both $10 \mathrm{mg}$ morphine po and placebo im, or an equivalent regularly dosed oral placebo (every four hours) with breakthrough pain medication consisting of oral placebo and $5-10 \mathrm{mg}$ morphine im. Subsequent to each request for breakthrough pain medication, the next regularly dosed oral solution was increased by $5 \mathrm{mg}$ (or equivalent volume of placebo) to a maximum of $40 \mathrm{mg}$ po $\mathrm{Q} 4 \mathrm{H}$. Time-averaged pain scores were lower on both postoperative day 1 and 2 in the group receiving regularly dosed morphine po $(P<0.05)$. Fewer pa. tients requested breakthrough pain medication on both days

\section{Key words}

PAIN: postoperative;

ANALGESICS: morphine;

SURGERY: orthopaedic, hip arthroplasty.

From the Faculty of Pharmaceutical Sciences,* University of British Columbia, Clinical Pharmacology, St. Paul's Hospital; $†$ and the Department of Anesthesia, $\ddagger$ at St. Paul's Hospital, University of British Columbia, Department of Statistics, $\S$ University of British Columbia.

Address correspondence to: Dr. James McCormack, Department of Pharmacy, St. Paul's Hospital, 1081 Burrard St., Vancouver, British Columbia, Canada V6Z 1 Y6.

Supported by a grant from the Vancouver Foundation, Vancouver, British Columbia, and the Pharmaceutical Manufacturers Association of Canada/Medical Research Council.

Accepted for publication 10th June, 1993. in the oral morphine group. The incidences of nausea and vomiting, and of decreased respiratory rates were similar in both groups. Regularly dosed oral morphine is inexpensive and should be compared to other methods of opioid delivery.

Une étude randomisée, contrôlée avec placébo et à double insu est réalisée dans le but de comparer l'uilisation de morphine po avec la morphine sur demande im chez 47 patients après une arthroplastie totale de la hanche. La randomisation est effectuées de façon à ce que les patient reçoñent: soit morphine $20 \mathrm{mg}$ po (dose initiale) répétée régulièrement (aux quatre heures) avec l'addition, pour les percées douloureuses sur demande de morphine $10 \mathrm{mg}$ po ou diun placébo im; soit un placébo oral administré avec la même régularité (aux quatre heures) avec l'addition pour les percées douloureuses de placébo po et morphine $5-10 \mathrm{mg} \mathrm{im}$. Subséquemment à chaque demande supplémentaires pour des percées douloureuses, la dose régulière suivante est augmentée de $5 \mathrm{mg}$ (ou d'un volume équivalent de placébo) jusqu'à un maximum de $40 \mathrm{mg}$ po aux quatres heures. Les scores d'évaluation moyens sont plus bas pour les deux premiers jours postopératoires pour le groupe qui reçoit de la morphine régulièrement $(P<0,05)$. Moins de patients reçoivent de la morphine pour des percées douloureuses pendant ces deux jours dans le groupe morphine po. Lïncidence des nausées et vomissements et de bradypnée est la même dans les deux groupes. La morphine administrée po régulièrement coûte peu et devrait être comparée aux autres méthodes d'administration.

Studies have shown that postsurgical pain (PSP) treatment is inadequate despite the availability of effective opioid analgesic agents. ${ }^{1}$ One reason for inadequate pain control appears to be the use of on-demand dosing of opioids.' This common form of treatment requires the patient to experience pain before an analgesic is given 
and thus sets up a cycle of pain relief followed by the return of pain which results in poor pain control. One reason for this on-demand or reactive approach is that the degree of PSP varies widely among patients, making it difficult to predict an individual patient's opioid requirements. For this reason, new methods of PSP pain treatment have been developed. Patient-controlled analgesia, continuous opioid infusions, and epidural administration of opioids appear to be more effective than ondemand opioid administration via im injection; however, these methods are expensive, require a specially trained clinical staff and also have specific adverse effects associated with their use. ${ }^{2-6}$ Patient-controlled analgesia is also not available at many institutions, and in institutions with patient-controlled analgesia programmes there is often not enough equipment or staff to provide this service to all postsurgical patients.

Regularly dosed po morphine (every four hours) has gained acceptance as the treatment of choice in patients with chronic cancer pain, but is almost never used to treat acute PSP. Oral morphine therapy is inexpensive, easy to titrate, and has high patient acceptance compared with im injection but there is reluctance to use this route immediately after surgery because of concerns about its absorption and effectiveness. In an unblinded study of paediatric orthopaedic patients, O'Hara et al. ${ }^{7}$ evaluated oral liquid morphine given on a regularly dosed basis for the treatment of PSP and found that more patients were pain-free with regularly dosed po morphine than with on-demand im meperidine. A number of studies ${ }^{8-13}$ have shown that the use of regularly dosed sustained release oral morphine (SRM) tablets is effective in patients with PSP. Unfortunately, some of these studies failed to give the oral preparation and/or to measure pain relief in the postsurgical period, which is the time when concern about the efficacy of po opioids exists. In addition, SRM preparations may not be appropriate for the treatment of acute PSP because they lack a rapid onset and ease of titration, which are key features required in a preparation being used for the treatment of acute pain lasting 24-72 hr.

In an unblinded pilot study, we recently observed ${ }^{14}$ that regularly dosed po morphine appeared to be effective and well tolerated for the treatment of pain following total hip arthroplasty. In this pilot study, only three of 13 patients required an increase in dose (from a starting dose of $20 \mathrm{mg}$ po $\mathrm{Q} 4 \mathrm{H}$ to $30 \mathrm{mg}$ po $\mathrm{Q} 4 \mathrm{H}$ ) and this dose was well tolerated. This suggested to us that a dose of $20 \mathrm{mg}$ po Q4H could be used as an initial dose in further trials.

Despite the concerns raised about the effectiveness of on-demand im morphine it continues to be a very common method for treating PSP. If regularly dosed po mor- phine could be shown to be safe and effective in the treatment of PSP it could then be compared with more complicated and costly methods of PSP management.

The trial reported here, following our pilot study of regularly dosed po morphine, was designed to compare, in a randomized controlled fashion, the efficacy and tolerability of regularly dosed po morphine, and conventional on-demand im morphine for the treatment of pain following total hip arthroplasty.

\section{Methods}

This study was approved by the ethics review boards at St. Paul's Hospital, Vancouver, B.C. and the University of British Columbia and all subjects gave written, informed consent. Subjects were recruited by approaching all patients who were scheduled to undergo total hip arthroplasty and receive general anaesthesia. The type of general anaesthesia was chosen by the attending anaesthetist. Patients were excluded if any of the following was present:

(a) marked COPD, asthma, congestive heart failure or unstable angina ( $\geq$ ASA III),

(b) a history of allergy to morphine or morphine-like opioids,

(c) an incomplete gastrointestinal tract or a gastrointestinal motility disorder.

All patients had their pain initially controlled with morphine $1-4 \mathrm{mg} i v$ as needed every five minutes while in the post-anaesthetic room (PAR) according to normal hospital procedure.

Upon arrival on the surgical ward, patients were assigned either to the experimental treatment group or to the control group, using a stratified randomization protocol. Patients were stratified according to sex, age $(<60$ or $\geq 60 \mathrm{yr}$ ) and arthritis status (presence or absence of rheumatoid arthritis). Within each of the eight strata, patients were randomized in blocks of four. Most doubleblind, randomized-control drug trials presume easy masking or disguise of the experimental and control groups that are to be compared. Typically, the experimental and the control medication (or placebo) can both be administered in tablets that are identical with respect to taste, appearance, etc. In the present trial, blinding patients and clinicians was more difficult since we wished to compare po morphine and im injection of morphine. To achieve blinding in this circumstance, our protocol required that all patients receive both regular $p o$ and on-demand im medications. For the experimental treatment group, the po medication, given regularly and on-demand, was morphine; and the im medication, given with the on-demand po medication, was placebo. The control group received po placebo and on-demand morphine injections.

On postoperative day (POD) 0 , each patient began to 
receive a regularly dosed $(\mathrm{Q} 4 \mathrm{H}$ at $0200,0600,1000,1400$, $1800,2200)$ oral solution containing either $20 \mathrm{mg}(5$ $\mathrm{mg} \cdot \mathrm{ml}^{-1}$ ) of morphine (for patients in the experimental treatment group) or an equivalent volume of similar tasting solution (for controls) from the time they arrived on the surgical ward from the PAR. All patients were informed that they could request and receive medication for breakthrough pain on-demand at any time during the study. Patients randomized to the experimental (regularly dosed po morphine) group were given morphine $10 \mathrm{mg}$ $p o$, plus im placebo when requested; while control patients (receiving regularly dosed placebo) received po placebo plus morphine $5-10 \mathrm{mg}$ im when requested, thus simulating on-demand im morphine. The dose of im morphine was determined by the surgeon involved in the case to allow us to mimic the practice at our hospital.

Subsequent to each request for breakthrough pain medication, the next regularly dosed oral solution was increased by $5 \mathrm{mg}$ (or an equivalent volume of placebo) to a maximum of $40 \mathrm{mg} \mathrm{Q} 4 \mathrm{H}$ (dose escalation). If pain control was still unsatisfactory, the investigators were to be contacted and further dosage adjustments would be made as deemed appropriate. The regularly dosed po solution was administered until 0200 on POD 3.

We recorded data on pain, medication, and adverse effects for each patient from the time of arrival on the surgical ward through two full POD's. The POD 1 included observations from 0200 of the morning after the operation through to 2200; and POD 2 observations corresponded to the next $0200-2200$ period.

Pain intensity was evaluated by the patient at fourhour intervals prior to each oral dose. At each assessment time patients were given a sheet of paper which contained a visual analogue scale (VAS) consisting of a vertical $10 \mathrm{~cm}$ line with the top of the line labelled "unbearable pain" and the bottom labelled "no pain." The patient was instructed to place a mark on the line which indicated the level of pain experienced at that time.

The level of sedation was assessed by the nurse before each dose using a four-point scale on which 1 indicated awake, 2 easily arousable, 3 difficult to arouse, and 4 unarousable. Respiratory rate (RR) was also recorded prior to each dose. If the patient scored either 3 or 4 on the sedation scale, or the respiratory rate was $<10$, then the dose was omitted. Any occurrences of nausea and vomiting were also recorded during the study period. Antinauseants were used at the discretion of the investigators. All patients received a stool softener (docusate $100 \mathrm{mg}$ po BID) and a stimulant laxative (bisacodyl 10 mg po every morning) during the study to help prevent any constipation that might occur either from the opioids or decreased ambulation during the postsurgical period.
TABLE I Patient Demographics

\begin{tabular}{lcc}
\hline & $\begin{array}{l}\text { Regularly dosed } \\
\text { po morphine }\end{array}$ & $\begin{array}{l}\text { On-demand } \\
\text { im morphine }\end{array}$ \\
\hline Male & 6 & 6 \\
Female & 17 & 18 \\
Number of patients & 23 & 24 \\
Mean age (yr) & 64 & 65 \\
\hline
\end{tabular}

\section{Data analysis}

Since patients completed surgery and returned to the surgical ward at different times during the day of surgery (POD 0), their oral medication was initiated at different times during the day. Therefore, before initiation of the study, it was decided to compare pain control only in the two groups on POD 1 and POD 2. Patients' level of pain may vary at different times during the day so we determined pain intensity every four hours. Group mean pain scores (sum of pain scores divided by the number of patients) at each time were calculated for both groups to assess visually the level of pain throughout the study. Since there is no simple nonparametric analogue to a repeated measures analysis of variance it was decided, before the study, that pain control in the two groups would be compared using a time-averaged pain score (TAPS) for each patient. Each patient's TAPS was calculated as the mean of the six VAS scores obtained on each POD 1 and 2. These values were used to compare treatment and control groups via a Wilcoxon ranksum test (Mann-Whitney test) with significance set at $P<0.05$ (one-sided). Also, the proportions of patients requesting breakthrough (on-demand) pain medication in the two groups were compared using Pearson's chi-square for a $2 \times 2$ table, with significance set at $P<0.05$. The proportions of patients experiencing particular adverse effects were similarly compared using Pearson's chisquare.

\section{Results}

A total of 47 patients were enrolled in the study and patient demographics are outlined in Table I. There were six withdrawals in the regularly dosed oral morphine group and seven in the on-demand intramuscular morphine group. The reasons for withdrawal are outlined in Table II. The data for these patients, up to the time of withdrawal, were included in the analysis (intention-totreat). The group mean pain scores, beginning at 1400 on POD 0 are plotted at four hour intervals in Figure 1 (due to variation in time to arrival on ward, the results at the 1400 time were for only 9 and 12 patients in the po morphine and im groups respectively). Group mean pain scores upon arrival on the ward were 6.2 and 4.6 for the morphine po and im groups respectively. The 


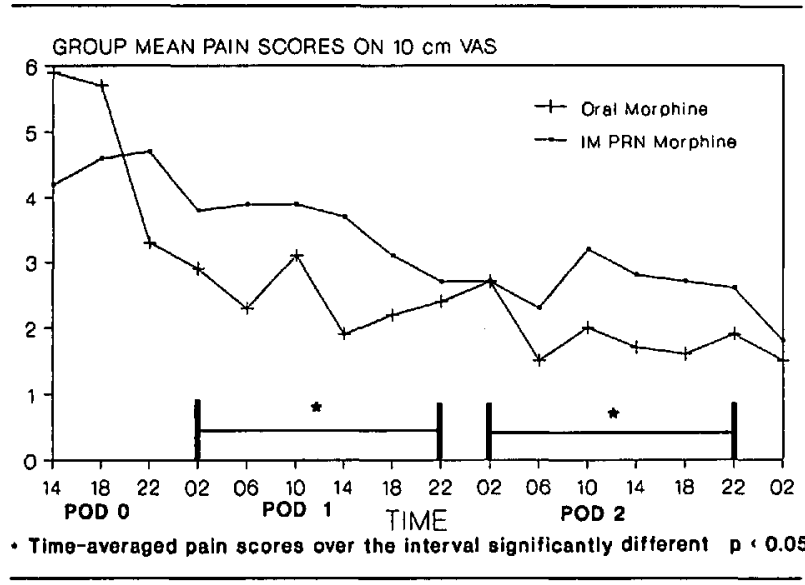

FIGURE 1 Group mean pain scores during postoperative day (POD) 0,1 , and 2 . Time $=$ time of day.

TABLE II Withdrawals (some patients counted twice if withdrew for more than one reason)

\begin{tabular}{lll}
\hline Reasons & $\begin{array}{l}\text { Regularly dosed } \\
\text { po morphine }\end{array}$ & $\begin{array}{l}\text { On-demand } \\
\text { im morphine }\end{array}$ \\
\hline Total number of patients & 6 & 7 \\
Nausea and vomiting & 2 & 4 \\
Poor pain control & 1 & 3 \\
Sedation & 3 & 1 \\
Apnoeic episodes & 0 & 1 \\
\hline
\end{tabular}

group mean pain scores show a continual reduction in the level of pain over the three-day period in each group. However, the mean pain scores in the experimental group were consistently lower than those in the control group. The time-averaged pain scores for POD 1 and POD 2 were lower in the po morphine group than in the group receiving on-demand im morphine $(P<0.05)$ using a two-sample rank-sum test. The proportion of patients requesting medication for breakthrough pain was also lower on both POD 1 and POD 2 in the oral morphine group (Figure 2). The mean number of requests for medication for breakthrough pain during the entire study period was 0.9 (Range $0-3$ ) and 2.3 (Range $0-10$ ) in the regularly dosed po morphine and the on-demand im morphine group respectively. In addition, 12 of 23 patients had no requests for breakthrough morphine in the po morphine group whereas only six of 24 patients in the on-demand im group had no requests during the whole study period $(P<0.02)$.

There was no difference in the incidences of adverse effects between groups (Table III).

\section{Discussion}

The results indicate that regularly dosed oral morphine was more effective than on-demand intramuscular mor-

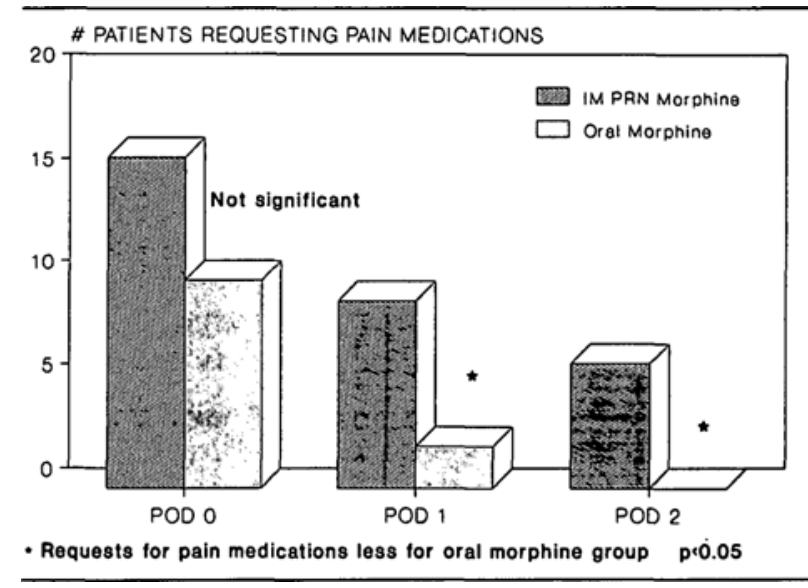

FIGURE 2 Patient requests for pain medications during postoperative day (POD) 0,1 , and 2 .

TABLE III Frequency of adverse effects

\begin{tabular}{lcc}
\hline & $\begin{array}{c}\text { Regularly dosed } \\
\text { po morphine }\end{array}$ & $\begin{array}{c}\text { On-demand } \\
\text { im morphine }\end{array}$ \\
\hline $\begin{array}{l}\text { Number of patients reporting } \\
\quad\end{array}$ & \\
$\quad$ at least one episode of & 13 & 16 \\
$\quad$ nausea & 31 & 28 \\
Total episodes of nausea & & \\
Number of patients reporting & 9 & 9 \\
$\quad$ at least one episode of & 16 & 18 \\
$\quad$ vorniting & 5 & 1 \\
Total episodes of vomiting & & 2 \\
Sedation (difficult to arouse) & 0 & \\
Episodes of respiratory rate & & \\
$\quad<10$ bpm & &
\end{tabular}

phine in treating patients' pain on POD's 1 and 2. The TAPS, which provides an indication of each patient's amount of daily pain was lower in the po morphine group. This result does not necessarily mean that there was a clinically important difference in the pain scores between the two groups. However, a clinical difference can be inferred from the difference in the frequency of on-demand requests for pain medications. The frequency of on-demand requests was lower in the po morphine group. However, in spite of the presence of PSP, as indicated by the decline in mean pain scores by the end of POD 3 and the effect of po morphine on the TAPS, there were relatively few on-demand requests in the $p o$ placebo group (mean 2.3). In our previous experience, patients undergoing total hip arthroplasty request an average of six intramuscular injections of opioid analgesics during the early postsurgical period. It is possible that the placebo effect reduced patients' perceived needs for on-demand therapy. This would not be surprising since it is known that placebo can provide good to excellent anal- 
gesia in $30-40 \%$ of patients with moderate to severe acute pain. ${ }^{15}$

The incidences of adverse effects were similar in both groups, although sedation may be more frequent in patients treated with po morphine and a larger study population might have detected such an effect to be significant.

One area of concern regarding the use of po medication after surgery is that nausea and vomiting will preclude or reduce their effectiveness. In the present study, nausea and vomiting rarely, if ever, occurred around the time of po opioid administration and did not preclude the use of po medication. The majority of postsurgical patients can tolerate sips of fluids and ice chips; and immediately after surgery clinicians routinely give most medications $p o$, with the exception of analgesics and antibiotics. Most patients, except those with nasogastric tubes, can use the po route for delivery of analgesics in the immediate postsurgical period. Despite this, however, many clinicians have concerns about the use of the po route immediately after surgery. ${ }^{16}$ While $10-37 \%$ of patients may exhibit episodes of nausea and vomiting after surgery it is usually transient and associated with ambulation or the ingestion of excessive amounts of fluid or food too soon after surgery and rarely prevents the patient from receiving po medications. Cohen et al., ${ }^{17}$ in a retrospective review of 112,721 patients after anaesthesia, found that while nausea and vomiting were the most common postsurgical complications, such episodes were greater than expected or required treatment in only $5 \%$ of patients.

Another common concern with the use of the po route immediately after surgery is the possibility of decreased gastrointestinal motility and decreased absorption of medication. ${ }^{16}$ While there is reason for concern that postsurgical gastric stasis may delay oral morphine absorption, only four studies have evaluated the effect of surgery on the absorption of oral morphine. ${ }^{11-13,18}$ In general, the authors of these studies concluded that the absorption of oral morphine is delayed in the postsurgical period. Unfortunately, all four studies evaluated absorption from sustained-release morphine (SRM) tablets, which exhibit very different absorption characteristics from liquid oral morphine. Also, these studies measured too few morphine concentrations in too few patients to obtain an accurate indication of the effect of surgery on oral morphine absorption. The poor absorption characteristics of the SRM may be due to the type of dosage being used. Solid particles leave the stomach more slowly than liquid preparations and the stomach usually retains particles that are larger than $1 \mathrm{~mm}$. Interestingly, fluid may leave the stomach despite the lack of gastric activity, which may allow the use of liquid analgesic preparations despite gas- tric contractions being reduced after surgery. Park et al. ${ }^{19}$ have demonstrated that, while a single im morphine injection inhibited gastric activity, a single dose of a SRM preparation caused only slight inhibition of gastric emptying. This suggests that morphine administered by the oral route may not increase gastric stasis. Even if gastric emptying is delayed somewhat, only the rate of absorption, not the extent of absorption, would be affected. If so, regular dosing would still produce a clinically effective steady-state serum concentration.

The final concern about the use of the oral route is that first-pass metabolism after oral administration leads to low bioavailability of orally administered opioids. ${ }^{16}$ While it is true that most orally administered opioids undergo extensive first-pass metabolism, this should not preclude their use in the treatment of PSP any more than it does in the treatment of chronic pain as long as patient-driven dose escalation is allowed. It is possible that slow absorption could increase metabolism to morphine-6-glucuronide which is effective as an analgesic. At present, there is no evidence to suggest that opioids undergo any more or less first-pass metabolism following surgery.

Our data indicate that regularly dosed oral morphine is effective in the treatment of PSP following total hip replacement surgery. Regularly dosed oral morphine was compared to intermittent intramuscular injections of morphine because this approach to the treatment of postsurgical pain is still very commonly used. We could have included a regularly dosed intramuscular group to our study, and this may have provided similar results as our regularly dosed oral morphine group. However, we wished to compare regularly dosed oral morphine with a treatment modality that is still commonly used. Regularly dosed im morphine is not commonly used and has low patient acceptance due to the need for repeated needle sticks. While some clinicians suggest that patientcontrolled analgesia is the gold standard for the treatment of PSP, many hospitals cannot afford to provide this service to any or all of their patients. For this reason intermittent im morphine is still a very common method of PSP treatment.

Oral morphine has many potential advantages over other methods of PSP treatment. It is easy to administer and titrate, has high patient acceptance, and is inexpensive. At present, the cost of administering regularly dosed oral morphine for $48 \mathrm{hr}$ after surgery is $\$ 0.89$ per patient (\$0.77 for 12 doses of oral morphine and \$0.12 for twelve medication administration cups) plus nursing time. With morphine usually being a wardstock item little pharmacy time is needed.

To ensure the safety of regularly dosed oral morphine for patients undergoing total hip arthroplasty, we recom- 
mend that respiratory rate and level of sedation be monitored in patients before each regularly scheduled oral morphine dose, as should be done prior to the administration of any opioid. If excess sedation or evidence of respiratory depression is observed, the regularly scheduled dose should be withheld. The use of regularly dosed oral morphine in the treatment of PSP cannot yet be extrapolated to patients undergoing surgery involving manipulation of the gastrointestinal tract as this may lead to reduced or delayed morphine absorption. The absorption pharmacokinetics of oral opioids, particularly in the first $24 \mathrm{hr}$ after surgery, must be evaluated before wholesale recommendations can be made about the use of the oral route for the treatment of PSP. In view of its potential advantages, regularly dosed oral morphine should be compared to other forms of opioid administration in patients with PSP secondary to orthopaedic procedures.

\section{Acknowledgments}

The authors wish to thank Rebecca Porter R.N., Shelley Dean R.N. and the Nurses on 9A and 9B, the orthopaedic surgeons, and the Pharmacy Department at St. Paul's Hospital for their diligent work.

\section{References}

1 Sriwatanakul K, Weis OF, Alloza JL, Kelvie W, Weintraub $M$, Lasagna $L$. Analysis of narcotic analgesic usage in the treatment of postoperative pain. JAMA 1983; 250: 926-9.

2 White $P F$. Use of patient-controlled analgesia for management of acute pain. JAMA 1988; 259: 243-7.

3 Rutter PC, Murphy F, Dudley HA. Morphine: controlled trial of different methods of administration for postoperative pain relief. BMJ 1980; 280: 12-3.

4 Church JJ. Continuous narcotic infusions for relief of postoperative pain. BMJ, 1979; 1: 977-9.

5 Eisenach JC, Grice SC, Dewan DM. Patient-controlled analgesia following cesarean section: a comparison with epidural and intramuscular narcotics. Anesthesiology 1988; 68: 444-8.

6 Stanley TH. Narcotics for acute postoperative pain. Is intramuscular administration passé? Postgrad Med 1983; 73: 107-14.

7 O'Hara M, McGrath PJ, D'Astous J, Vair CA. Oral morphine versus injected meperidine (Demerol) for pain relief in children after orthopedic surgery. J. Pediatr Orthop 1987; 7:78-82.

8 Watson D. Pain relief following thoracotomy. In: Wilkes E, Levy $\mathbf{J}$ (Eds.). Advances in Morphine Therapy. Royal Society of Medicine International Congress and Symposium Series 1983; 64: 37-41.

9 Kimberley A. A comparison of oral slow-release morphine sulphate with intramuscular papaveretum for analgesia following in-patient dental surgery. I $n$ : Wilkes E, Levy J
(Eds.). Advances in Morphine Therapy. Royal Society of Medicine International Congress and Symposium Series 1983; 64: 47-50.

10 Derbyshire DR, Vater M, Maile CI, Larsson IM, Aitkenhead $A R$, Smith $G$. Non-parenteral postoperative analgesia. A comparison of sublingual buprenorphine and morphine (slow release) tablets. Anaesthesia 1984; 39: 324-8.

11 Derbyshire DR, Bell A, Parry PA, Smith G. Morphine sulphate slow release. Comparison with i.m. morphine for postoperative analgesia. $\mathrm{Br} \mathrm{J}$ Anaesth 1985; 57: 858-65.

12 Banning AM, Schmidt JF, Chraemmer-Jörgensen B, Risbo $A$. Comparison of oral controlled release morphine and epidural morphine in the management of postoperative pain. Anesth Analg 1986; 65: 385-8.

13 Pinnock CA, Derbyshire DR, Achola KJ, Smith G. Absorption of controlled release morphine sulphate in the immediate postoperative period. Br J Anaesth 1986; 58: 868-71.

14 McCormack JP, Warriner CB, Levine M, Forster-Coull J. Regularly scheduled oral morphine for post surgical orthopedic pain. Canadian Journal of Hospital Pharmacy 1992; 45: $101-5$.

15 Spiro HM. Doctors, Patients and Placebos. New Haven: Yale University Press, 1986: 86-7.

16 Mitchell $R W D$, Smith $G$. The control of acute postoperative pain. Br J Anaesth 1989; 63: 147-58.

17 Cohen MM, Duncan PG, Pope WDB, Wolkenstein C. A survey of 112,000 anaesthetics at one teaching hospital (1975-83). Can Anaesth Soc J 1986; 33; 22-31.

18 Lew JK, Mobley KA, Achola KJ, Horne A, Smith G. Postoperative absorption of controlled-release morphine sulphate. A study in patients given no parenteral opioids. Anaesthesia 1989; 44: 101-3.

19 Park GR, Weir D. A comparison of the effect of oral controlled release morphine and intramuscular morphine on gastric emptying. Anaesthesia 1984; 39: 645-8. 